\title{
Methodology of Reverse Engineering Implemented in the Process of Digitalization and Conservation of Wooden Carvings
}

\author{
Atanas Kochov*, Lidija Stoleska
}

\begin{abstract}
The purpose of this paper is to present the benefits of reverse engineering when applied to cultural heritage i.e. digitalizing and duplicating an artwork in lack of original documentation, drawings, or computer models; Usage of different methods for 3D scanning, reverse engineering softwares with a smart combination of history-based CAD with 3D scan data processing, for preparing the obtained 3D model for future production use; Describing tools and machines for rapid prototyping, materials used and the feasibility of its completion; Set of tools that provide opportunities for new projects and innovations but are also of cultural and historical significance.
\end{abstract}

Keywords: 3D scanning; digitalizing; preservation; rapid prototyping; reverse engineering

\section{INTRODUCTION}

The rapid development of science and technology, the application of new modern technologies, in every scientific and applied field, leads to the setting of new higher requirements related to the application of advanced technologies in designing production processes i.e. new approaches in new product development processes. The essence is to place a high quality product on the market. In such conditions, new technologies such as: rapid prototype technology (RP), rapid tool making technology (RT), reversible engineering (RE), and appropriate 3D scanning, 3D printing, are new and efficient technologies that provide companies with the opportunity to significantly reduce the time of product development and its placement on the market. [1] But, what is very important is that these new techniques and technologies are increasingly being used in the processes of protection of cultural heritage. Namely, Reversible Engineering is the process of replicating an existing object, assembly or product without having original drawings, documentation or computer models. [2] In this thesis, the focus is on the importance of reversible engineering and consequently on the appropriate tools, programs and machines in order to collect data on works of art, cultural heritage and wood carvings that have not been software stored, digitalized and stored in databases, and then be able to be rebuilt into physical models. We are witnessing frequent weather disasters, destruction by various factors, which can permanently destroy an object. Handmade woodwork becomes timeless if digitilized. When the necessary methodologies and methods are studied, an opportunity is created for making copies of them. The topic of scanning works of art is not current in Macedonia, so it leaves a lot of room for further upgrading. The aim of the research is to create a methodology of reversible engineering for digitalization of cultural heritage and new works in the field of wood carving art, as well as a practical part of the application of reversible engineering and visualization techniques through specific examples. All technologies that can lead to digitalization and reprocessing in 3D model have been developed. The aim is to specify the steps for obtaining a digitalized database and ways to create copies of wood carvings.

\section{DEFINING}

Art creators convert their ideas into physical models with different materials - like clay, wood, plaster, or foam - but a CAD model is necessary if the part needs to be manufactured. 3D scanners capture the basis of artistically inspired and man-made figures and precise records of original pieces for archiving or creating reproductions by using combining technologies. Another option to 3D scanning is by camera through photography (photogrammetry). Smartphones and smart technologies can easily and simply help preserve world heritage by digitalizing pre-built works of art and placing them in a database. Smart technologies are widely used across various industries, because they offer quality services in a short period of time. Its usage in cultural activities it's still not that developed and has space for improvement. The concept is shown in Fig. 1.

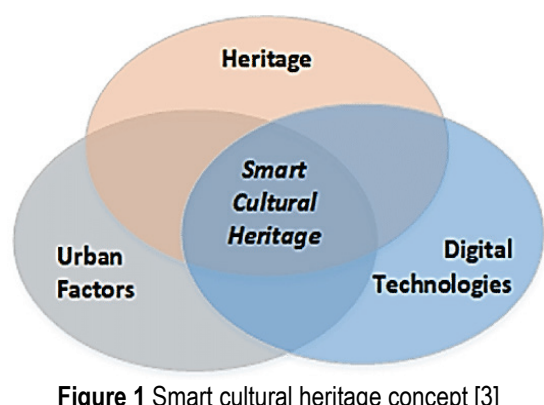

Quick, free and inexpensive smartphone applications can help you efficiently create digitalized objects [6] that can be stored on a server for later use.

For this purpose, except the objects to be scanned, the 3D scanner or camera, we need a professional reverse engineering software with a smart combination of historybased CAD with 3D scan data processing, for preparing the obtained 3D model for future production use. In the current case, the Geomagic Design X and Autodesk Recap programs were used.

Another programs that should be taken into consideration are Virtual CNC simulations of machining 
processes, so that an object can be manufactured or duplicated (carving, for current case study) with minimum mistakes, so no additional costs happen and sustainability is kept on a high level. Powerful numerical controls are required for complex machine technologies. Programming part of the machine is hard and time consuming. However, with the usage of simulation and virtual commissioning there are possibilities available that support the manufacturer in ways that can reduce time and costs. Such methods are nowadays recognized under the term "Digital Twin".

There are no end to the usage and purposes of the $3 \mathrm{D}$ scanners, smartphone applications and CAD/CAM programs which everyday are getting better and more useful in different areas. The promotion of cultural heritage can be developed through implementation of certain knowledges, technologies and social inclusion. As result, with participation and promotion of each, smart cultural heritage can be built.

Steps of producing a prototype of a previously digitalized wooden carving will be presented through practical example. Prototyping with $\mathrm{CNC}$ machines (CNC wood routers) and 3D printing will be explained.

\section{TECHNOLOGIES AND METHODS APPLIED}

The steps that can be taken to reproduce an object will be briefly explained as well as comparing the needed technologies and their limitations.

\subsection{D Scanning}

$3 \mathrm{D}$ scanning is a technique of using a $3 \mathrm{D}$ scanner to capture shapes of objects. After 3D scanning, 3-dimensional files are made. With the help of $3 \mathrm{D}$ printers, those $3 \mathrm{D}$ files can be saved, edited and printed. There are different methods of 3D scanning based on different principles. Each of these methods have their own advantages, disadvantages and cost differences. 3D scans are compatible with CAD (Computer Aided Design) softwares and with 3D printing. When analysing reverse-engineering processes, 3D scans are the primary step for getting information about the object's design. One of the biggest obstacles to encounter when converting physical objects to digital is a major incompatibility between two different types of 3D models: meshes and solids. A 3D scanner outputs a mesh, rather than a constructive "solid" model. Meshes need to be reverse engineered to be made editable.

There are different types of 3D scanning methods: Laser pulse-based 3D scanning technology, Laser triangulation 3D scanning technology, Structured light 3D scanning technology, Photogrammetry, Contact-based 3D scanning technology.

\subsection{Scan to Model Programs 3.2.1 Geomagic for SolidWorks}

The most complete integrated model solution (Scan-toSolidWorks). It reduces the time required to build complex 3D models of objects by directly scanning or importing scan data into SolidWorks. Automated and advanced wizards quickly and easily create precise sketches, surfaces or feature-based editable solid parts inside SolidWorks. Geomagic for SolidWorks is a software toolset that plugs directly into a SolidWorks environment giving advanced capabilities to make tools point clouds and polygons more usable in the design process. This software supports a range of popular 3D scanners as well as the import of standard point and polygon file formats. Data can be imported or scanned directly in SolidWorks and the feature extraction wizards can be used to extract CAD sketches, surfaces and solids.

\subsubsection{Autodesk's ReCap Software}

This software allows direct point cloud opening. The technology lends itself readily to mechanical and manufacturing industries. Reality captures can be done of desired objects, for which design parameters aren't known. With this technology, you can overlay your new part to match the size, bolt-hole placement, etc. with exacting tolerances, in short period of time.

\subsection{Rapid Prototyping}

The process of creating prototypes quickly to visually and functionally evaluate an engineering product design.

When explaining fast prototyping, it is the rapid creation of a product that will be representative of the technology used and the precision of the desired design. Confirms the way of manufacturing, how the system works in reality. Multiple technologies can be used. When discussing the creation of a new product or design on the market, one cannot fail to include rapid prototyping as an integral part of the process. Rapid prototyping can be used at any stage of the product development cycle or for any component or sub-component and can be repeated numerous times along the new product design process. [4]

Fig. 2 shows the rapid prototyping process.

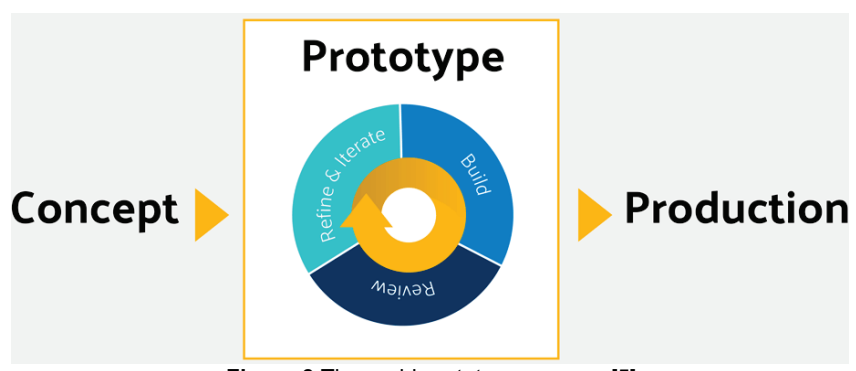

Figure 2 The rapid prototype process [5]

Rapid prototyping is not limited to one process. Many manufacturing techniques can be used to assemble a prototype.

Following are some of the types of rapid prototyping technologies divided in two groups:

- Additive manufacturing:

- $\quad$ Direct metal laser sintering(DMLS)

- $\quad$ Fused Deposition Modelling (FDM)

- $\quad$ Stereolithography (SLA) 
- $\quad$ Selective laser sintering (SLS)

- Other techniques:

- CNC Machining Prototyping

- Vacuum casting

- Investment casting.

\subsection{Comparison between Additive Manufacturing and CNC Machining Prototyping}

If we compare the two groups of technologies, we will quickly and easily see the difference. Namely, fast prototyping can be divided into subtraction technology and addition technology. The first group, subtraction prototyping, is designing the desired object from a certain material by machine engraving (taking off of block material). [6]

Additive manufacturing is opposite process. Structures are made by the addition of thousands of minuscule layers which combine to create an object. The process must be supported by a special CAD software which relays messages to the printer so it "prints" in the previously drawn or scanned figures. Suitable for use with a range of different materials, the cartridge is loaded with the filament for different purposes and later it's "printed" into the shape, layer by layer, fused together until the shape is done.

There are machines with different axis manipulation for manufacturing but the focus will be put on 5 -axis machining because of the complexity of the wooden carvings if it were to be reproduced. 5-axis machining involves using a $\mathrm{CNC}$ to move a part or cutting tool along five different axes simultaneously. Five axes machines are complex. Therefore, trained staff is needed to manage them, accompanied by appropriate CAD/CAM software for effective, efficient machine use, and error minimization.

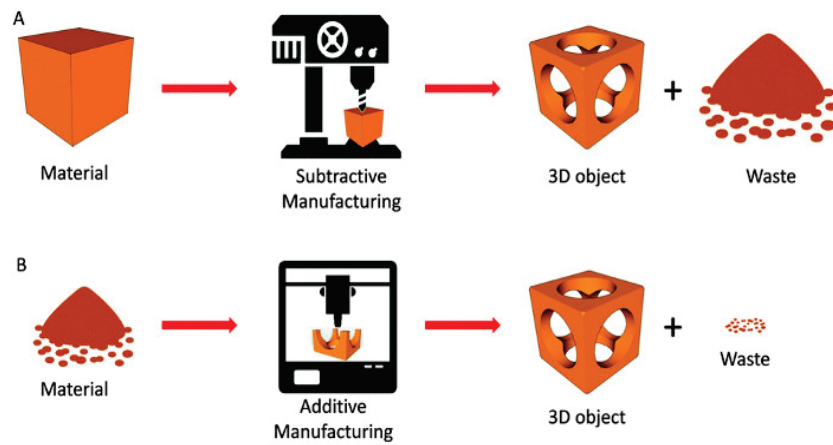

Figure 3 Visual difference between additive and subtractive manufacturing [6]

Fig. 3 graphically shows the difference between the technologies. The controller in the machine has to be able to process the data fast enough so the toolpath is a nice, smooth, uniform motion. For minimizing errors $\mathrm{CNC}$ simulation is the best tool. It is used to visualize virtual production sequences in machine tools in a realistic way. By mapping the complete working area of the machine (including all fixtures, tools and workpieces) and the movements of the machine (including visualization of the tool path and material loading and unloading) in $3 \mathrm{D}$, to the user can evaluate and optimize the process before the $\mathrm{CNC}$ programs are transferred to the machine. Predator Virtual $\mathrm{CNC}$ is an example of that kind of software designed to simulate and verify operation of your CNC machines on your PC.

For the current case study wood router ought to be used for production. It is controlled in the same way as a metal mill, but the CAD/CAM programs are more specific, such as Artcam, Mastercam, Bobcad, and AlphaCam. Wood routers are frequently used to machine other soft materials such as plastics. The advantages of $\mathrm{CNC}$ wood router (compared to general machine) as follows:

- High degree of automation

- Consistent quality

- High productivity

- Processing complex shapes

- Easy to implement CAD/CAM.

\subsection{Limitations of Technologies}

Each of the 3D scanning technologies has its advantages and disadvantages. The laser triangulation technology has advantage of accuracy and high resolution while the disadvantage is it is sensitive to the properties of the surface that it scans. Laser-pulse based technology is great for scanning large objects and environments, on the contrary, the main disadvantage with structured lighting 3D scanning is its sensibility to lighting conditions and therefore it's difficult to work outside. About photogrammetry, its main advantages are precision, acquisition speed and its capability of reconstructing subjects of various scales that are photographed from different perspectives.

When comparing the rapid prototyping technologies a few things are taken into consideration such as: used materials, precision, speed, waste and price. CNC mills can work on a huge variety of materials: metal alloys (e.g. aluminium, steel alloys, copper, and brass), hardwoods and softwoods, thermoplastics, acrylic, modelling foams etc. Variety of cutting tools for different materials are required, but tool-to-machine interfaces are commonly standardized so the tools can easily be exchanged. CNC mills/routers can be utilized to manufacture prototypes in the same material that will be used for the final product. Desktop 3D printers are usually restricted to a few materials, typically thermoplastics (PLA, ABS, sometimes nylon) or resins. Thermoplastics can be mixed with other materials such as ceramics, wood, metal, but the workpieces produced on a 3D printer are not as durable as produced on a CNC machine, and are brittle when subjected to higher pressures.

Thermoplastics and resin 3D printers use completely different methods, a resin printer cannot handle thermoplastics - and vice versa. CNC mills offer positioning accuracies of around $0.025 \mathrm{~mm}$ and tolerances of $0.0127 \mathrm{~mm}$. There are 3D printers supporting resolutions of $0.025 \mathrm{~mm}$ and $0.02 \mathrm{~mm}$. Comparing speed is difficult as $\mathrm{CNC}$ mills and 3D printers are typically used for different workpieces and materials. Different rapid prototyping technologies are used for different purposes. It is therefore difficult to compare specifications, such as processing speed. Roughly speaking, for larger objects the 3D printer takes many hours while the $\mathrm{CNC}$ machine can process an object in up to an hour. $\mathrm{CNC}$ mills are typically faster when chipping away material from 
a solid block than 3D printers that build objects layer by layer and occasionally have to slow down to avoid printing problems. The result: But, because it is a subtractive technology, CNC definitely produces more waste than $3 \mathrm{D}$ printing.

\section{MATERIALS}

Now that technologies were explained, next point would be implementing them into the subject of reproducing wooden carvings. It is known that wood is processed on CNC machines, but the first question when combining wood with additive manufacturing is: "How can you 3D print wood?" It is possible in a way, by printing with wood filament.

The material is mainly PLA that contains wood fiber. Tissue comparison of natural and printed wood is shown in Fig. 4. Objects printed with wood filament look wood-like. There are different versions of wood filament, depending on the manufacturer. There are potential difficulties when printing with wood filament. It is considered a "temperamental" material. Typically, wood filament is composed of around 70\% PLA and 30\% wood fiber. [7] Because of the added wood, it tends to be more delicate than regular PLA, breaking more easily. Early wood filaments were made with sawdust, but the final prints looked more like cardboard than wood. The advantage is its greater flexibility, but with today's wood fiber filaments, 3D printed objects can look, feel, and smell very much like carved wood. In combination with $3 \mathrm{D}$ printing these materials can help with preserving handmade wood designs.

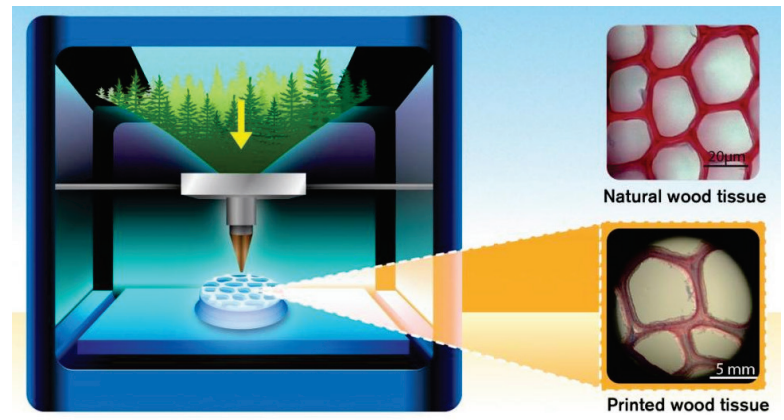

Figure 4 Comparison of natural wood tissue and printed wood tissue [7]

\section{PRACTICAL EXAMPLES}

The first carving chosen to be digitalized is part of a Bishop's throne. It has a vase shape, symbolically suggesting a crown worn by bishops in Byzantine style. [8] Second carving is a bird on a leaf, which is a part of an iconostasis as decoration. The third carving is head of a pillar also serving as a decoration on the top of an iconostasis. They represent an idea for the restoration of one of the first such cultivations that date back to the 5-6 $6^{\text {th }}$ century. Wood as a material for the processing of such works has a limited durability, is subject to aging, pest attacks unlike those made in stone and marble that have a much longer lifespan. The historical events of the last few years, the loss of certain buildings of cultural and historical significance such as the fire in Bigorski Monastery or the Officer's Home in Skopje and the development of digital and machine technology, all indicated the need to think about how digital records of valuable artwork can be provided that could then be used for restoration. The number of craftsmen and artists making such works is diminishing and the technical aids and knowledge of those used at the time are diminishing. Therefore, helping machine-made copy of a work of art (or a whole) is invaluable to the collective memory of national wealth and the preservation of world cultural heritage. The objects are displayed in Fig. 5, Fig. 6 and Fig. 7.

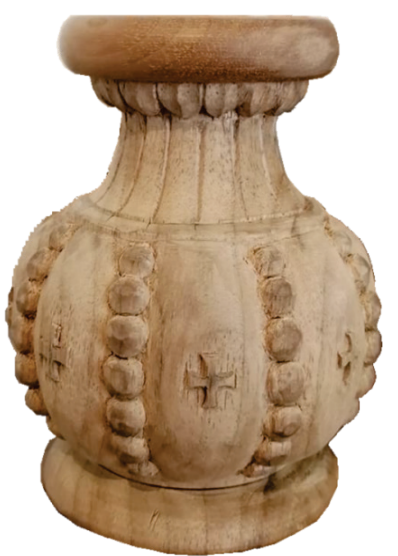

Figure 5 Small vase-like pedestal

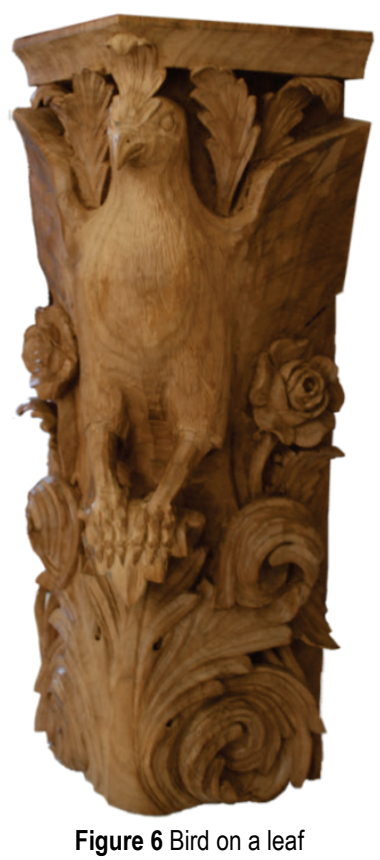

A 3D scanner was used on the principle of structured light 3D scanning technology to digitalize the first object, along with the GeoMagic program. For the other two carvings, a camera will be used on the principle of photogrammetry together with the AutoDesk Recap program. The NextEngine Scanner is a desktop 3D scanner that uses an array of lasers to scan objects at resolutions of $0.127 \mathrm{~mm}$, [9] shown in Fig. 8. 


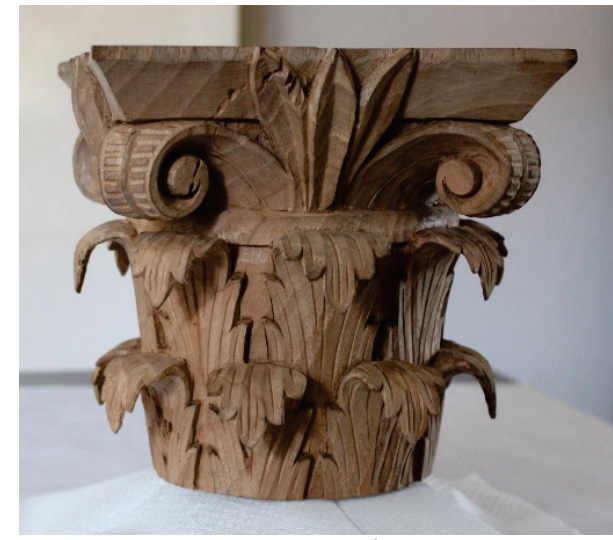

Figure 7 Head of pillar

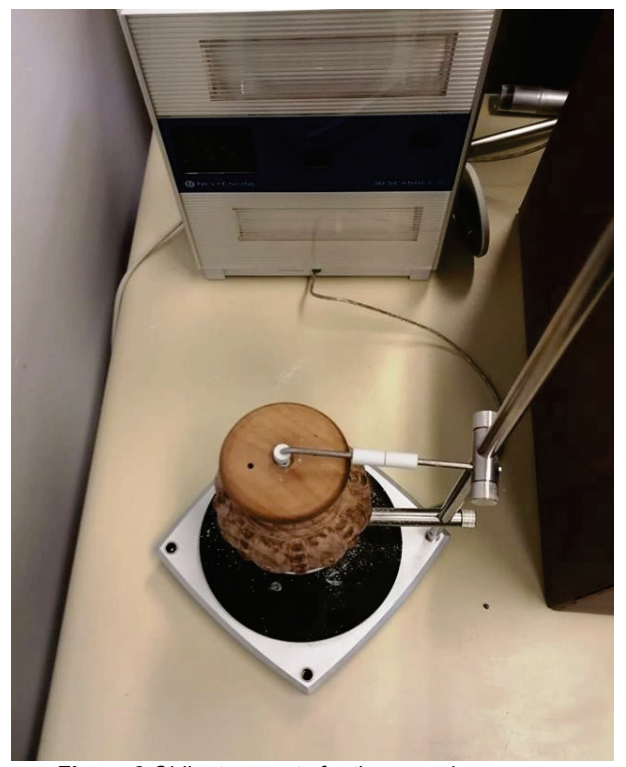

Figure 8 Obligatory parts for the scanning process

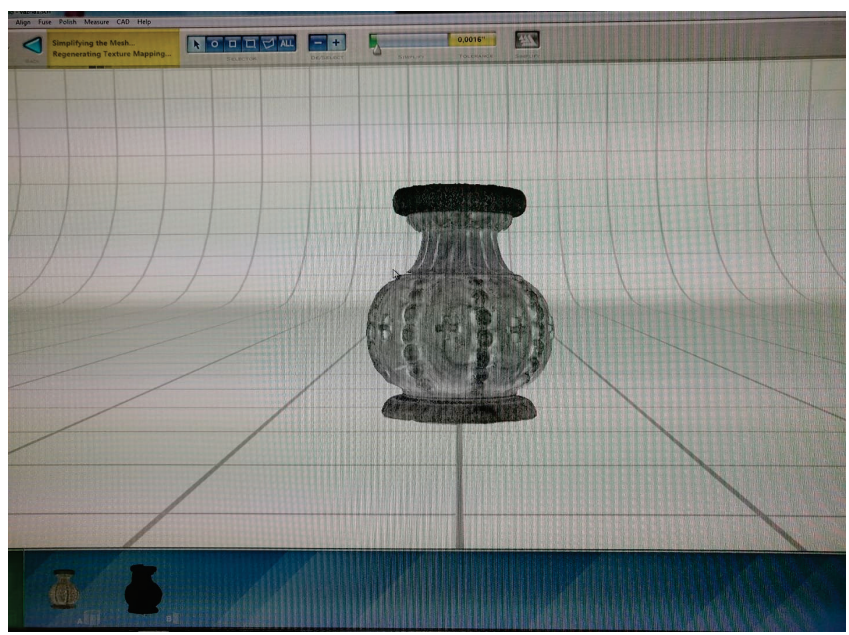

Figure 9 Finished scan of the carving

In Fig. 9 below, the finished scanning process of the vase-looking pedestal can be seen. This chosen carving needs little bit of cleaning after scanning. The software itself gives that option but the part still needs to be cleaned again in another program so that it can be used in future processing like 3D printing or $\mathrm{CNC}$ machining. It can easily be imported in SolidWorks or directly in 3D printer as STL file.

Fig. 10 shows the digitalized carving mesh cleaning, transferred in SolidWorks.

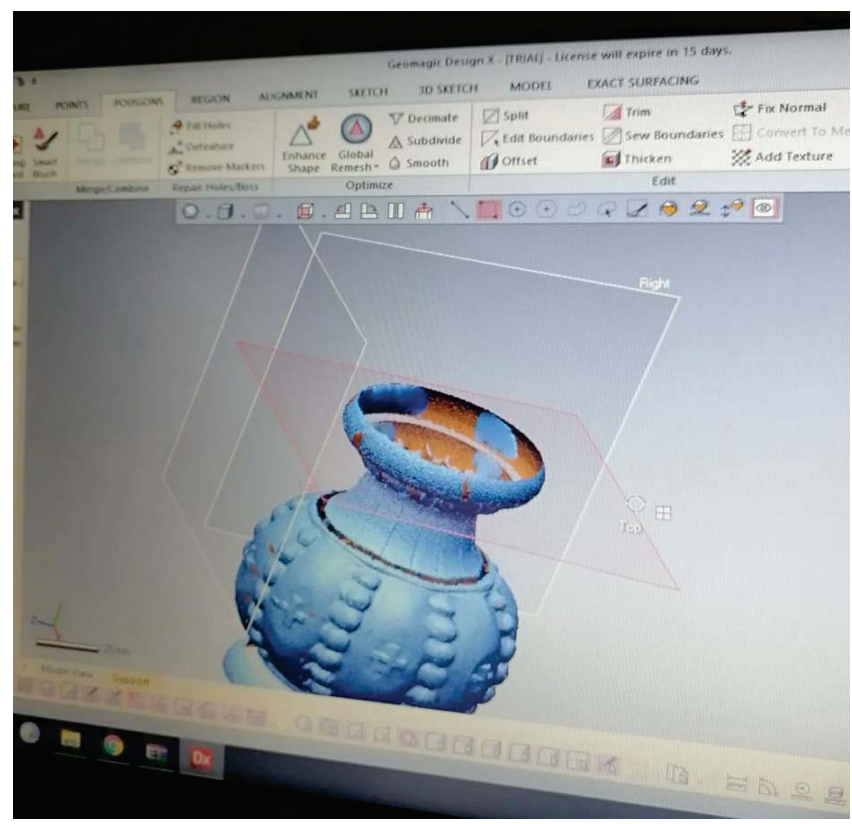

Figure 10 Mesh cleaning in GeoMagic

The following Figs. (11)-(16) show the use of photogrammetry for digitalizing the previously mentioned carvings. The digitalization process is as follows: Get the necessary camera images, clean the objects in AutoDesk Recap and import them as STL files. The STL file format has become the Rapid Prototyping industry's defacto standard data transmission format. [10]

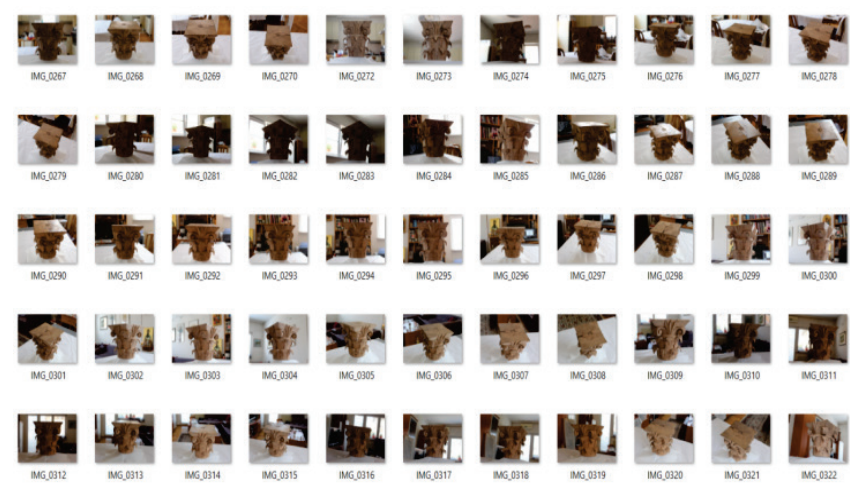

Figure 11 Required photos for photogrammetry completion

Fig. 12 shows the final product of AutoDesk Recap's photogrammetry.

Fig. 13 shows another possibility of AutoDesk Recap video of scanned object rotation.

Fig. 14 is a triangulate representation of the mesh (carving) and Fig. 15 is the object transferred in SolidWorks as solid body. 


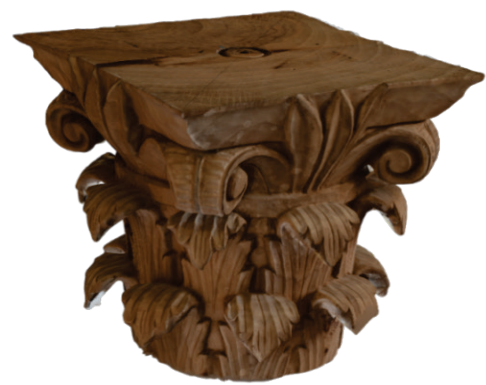

Figure 12 Head of pillar in RCM file

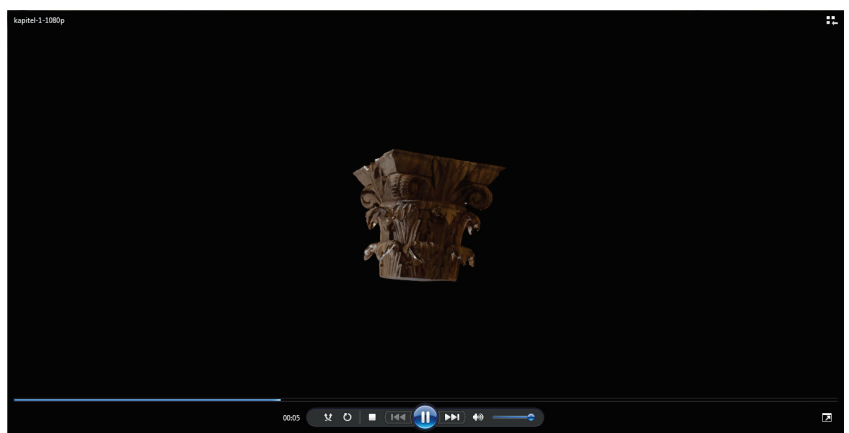

Figure 13 Video screenshot of the head of the pillar - Autodesk Recap possibilities

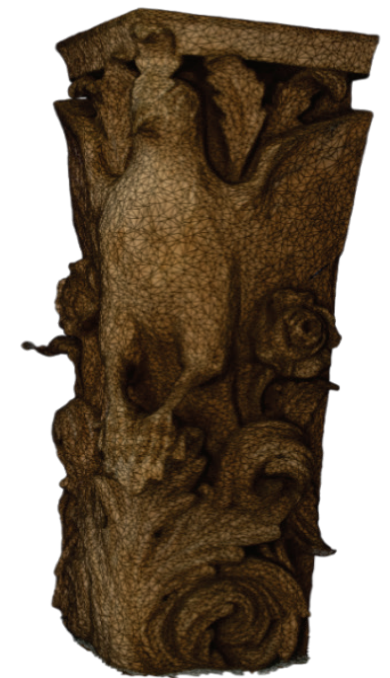

Figure 14 Triangulate representation of the mesh (carving)

Physical representation of the head of pillar was made (Fig. 17) on an Ender3 3D printer. Using Ultimaker Cura which is one of the most popular 3D printing softwares, the print was prepared with a few clicks. The software is integrated with CAD software for an easier workflow. Fig. 16 below, shows the setting up of the head of pillar before printing.

Printed with PLA (as the conditions allowed), but serving as proof of a possible reproducing when having the right printer and filament. Printing PLA is closest to wood filament.

Because of lack of suitable 5-axis machine, physical representation of a carving produced by wood router won't be shown. Manufacturing carvings on a $\mathrm{CNC}$ machine has many advantages, but it is very important to know that more research is needed than just buying the latest machine. Precise programming and good familiarity with tool tolerances are required.

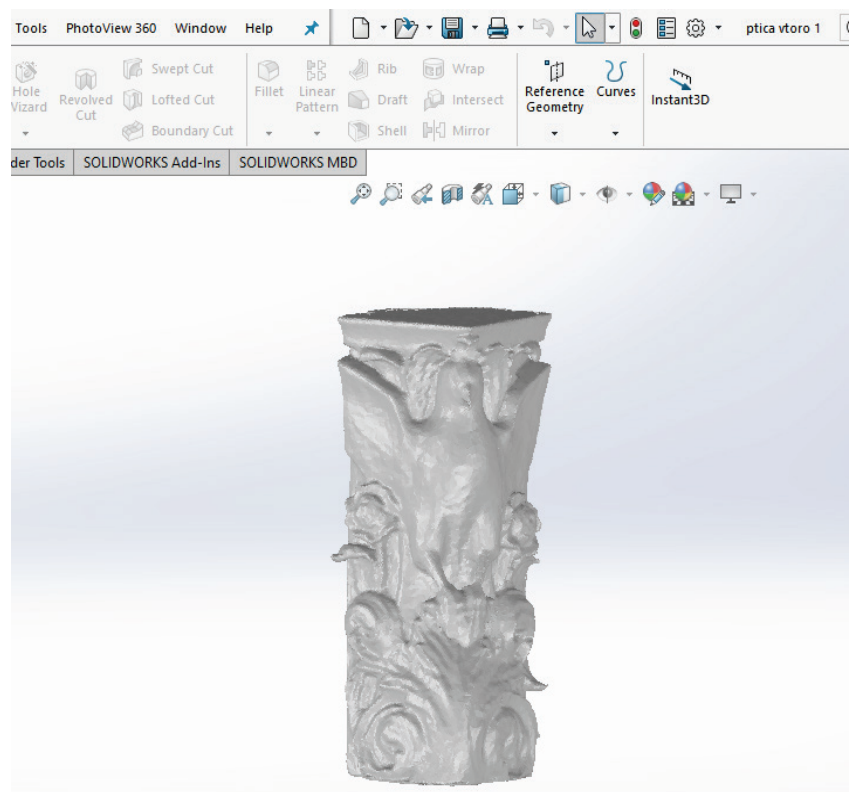

Figure 15 Solid body of the bird carving in SolidWorks

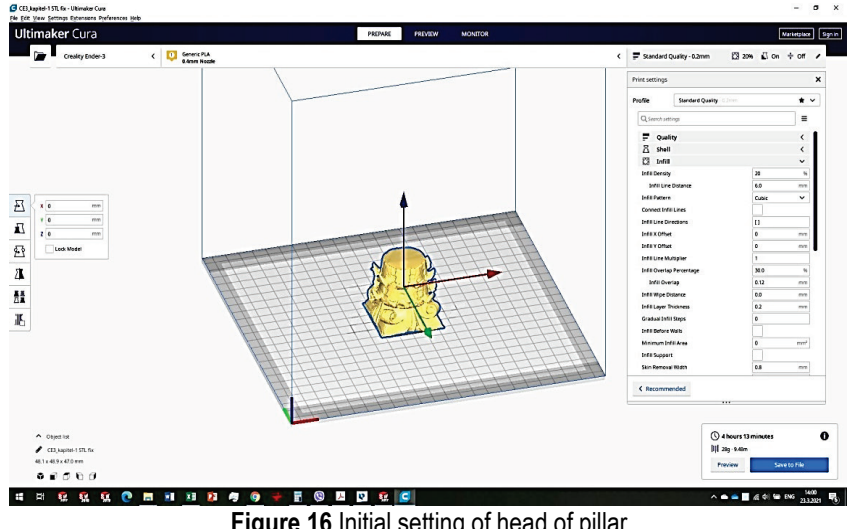

Figure 16 Initial setting of head of pillar

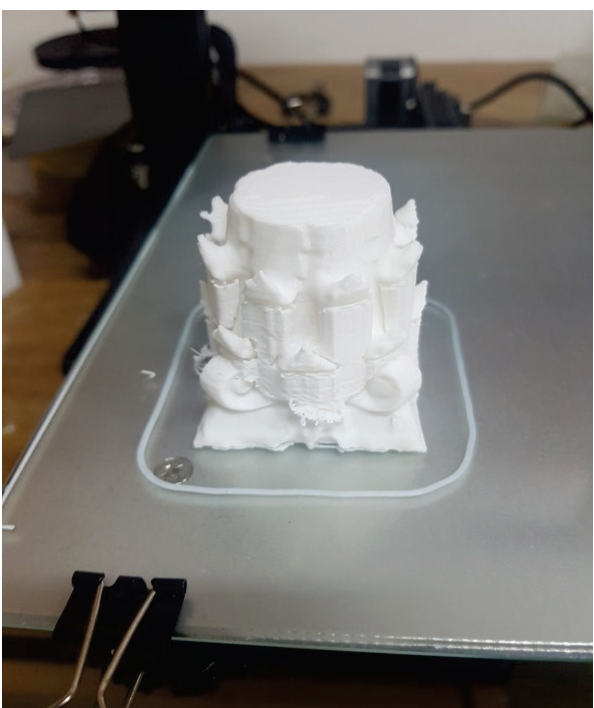

Figure 17 Final 3D print of the head of pillar 
Last but not least, smartphone usage for scanning is presented. The Qlone application was used for the purpose of this thesis. [11] A compatible mat is required to put an object on (carved cup-holder, Fig. 18), scanning can be continued. Pieces can be put horizontally or vertically.

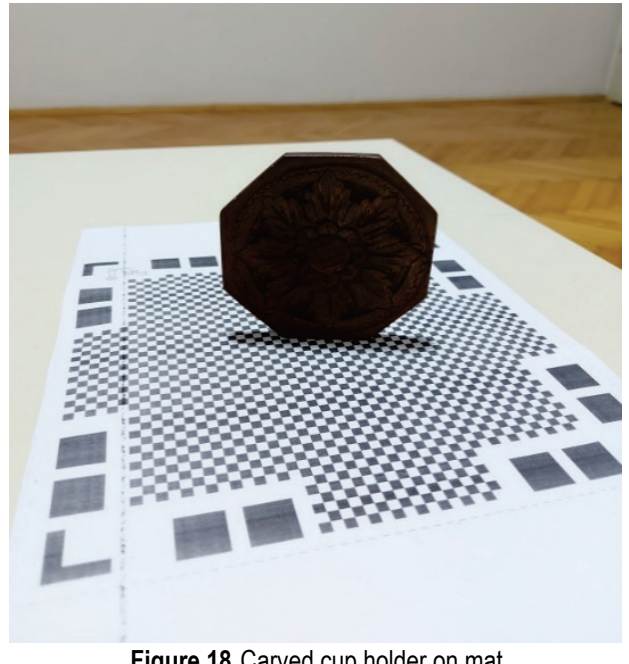

Figure 18 Carved cup holder on mat

Scanning of the carving will be complete after rotating with the smartphone's camera around it until every blue part of the hemisphere is gone (Fig. 19). In Fig. 20 the finished scan of the carver cup holder can be seen.

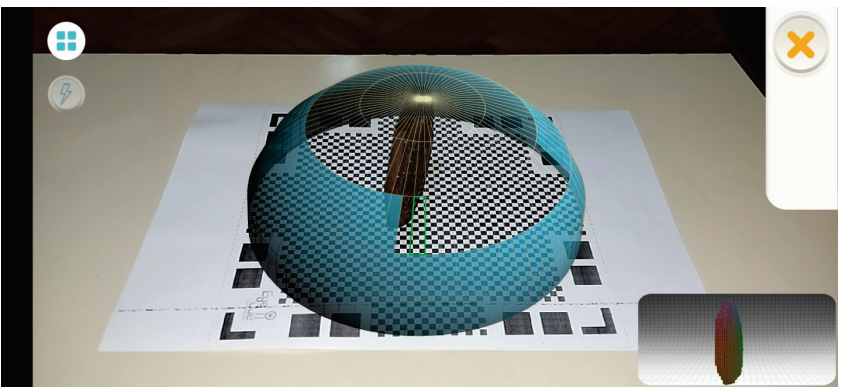

Figure 19 Blue hemisphere around carved cup holder

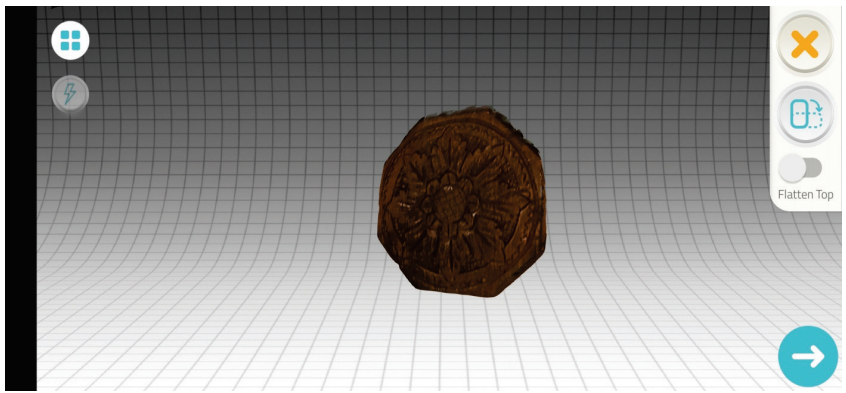

Figure 20 3D scanned carved cup holder

\section{COST OF INNOVATION}

So, how feasible is making business out of preserving works of art? You need the right equipment, machines and programs as well as a trained person for each of them to implement it. The basic and most important thing is to have a suitable scanner or camera so that we can digitalize carvings. Then, appropriate licensed programs are needed that will generate the required file and where an object can be 'cleaned'. Next, a 3D printer is needed or a CNC machine to get a physical model. But within all, there are a lot of additional costs, such as material, cleaning of excess material, space, electricity, maintenance, etc. You need to calculate the time and effort put to do the work, as well as to return your investment for equipment.

According to The Williamstown Art Conservation Centre, "Conservation cost is based on time and materials required, not on the value of the object". It is important to note that you are paying for a professional's time and expertise, so the rates for conservation range broadly based on the work's medium, age, cultural or historical significance, as well the methods required for conservation. [12]

\subsection{Cost of Equipment and Material}

3D printer materials and operating costs usually tend to be lower than those encountered when using traditional methods. The first thought that might come across mind is that printing an extremely detailed $3 \mathrm{D}$ model is very expensive - but that is not the case. It is shown that the biggest contributor to the price is the amount of material used and not its particular features.

CNC routers have many advantages over traditional hand tools with speed and automation being the greatest. This means work can be accomplished in a much faster pace, with a lot less employees - which on the other hand, saves money and time.

Depending on the complexity of the machine and what can be achieved with it, price ranges are as follows:

- $\$ 5,000-\$ 10,000$ for the most basic of three-axis

- $\$ 25,000-\$ 50,000$ for mid-range machines (used for panel processing and signage)

- $\% 50,000-\$ 150,000$ for high end machines.

Aside from machine costs, there are several other costs that need to be considered:

- Purchasing a computer aided (CAD) software package used for creating the design. Several option are available with prices anywhere from $\$ 2,500-\$ 10,000$.

- Training, depending on the familiarity level of staff, anywhere from $\$ 500-\$ 1,000$

- Machine installation, $\$ 500-\$ 1,000 /$ day

- Shipping, anywhere from several hundred dollars to $\$ 3,000$.

- 3D printer.

Prices for 3D printers can vary, with the cheapest being around $\$ 200$, average consumer type - around $\$ 700$ to several thousand dollars for high-end consumer printers. [13] It is fair to say that the cost for $3 \mathrm{D}$ printers has significantly dropped over the past few years with several high-quality options available from $\$ 1000$.

Three factors were considered when ranking the best 3D scanners: 
- Scan quality, price-performance ratio and customer feedback on the scanners. They can be grouped into three price ranges: cheap.

- Under $\$ 1,000$, medium priced - under $\$ 10,000$ and industrial - over $\$ 10,000$.

\section{CONCLUSIONS}

Set of knowledge, methods, methodologies, technologies and interests are assets to mechanical engineering as an assembly in helping to preserve a piece of art. It is important to note that the objects used throughout the case study, were not damaged. If all the possibilities offered by different professions are taken into consideration for conservation and restoration of art objects, we would have permanently preserved wealth. We leave that synergy to future projects. Each of those assets represent different areas of implementation, depending on the condition, form, function and intended use of the cultural item. Scientific methodologies engaged in the analysis of materials and ways of digitalization and processing are combined with humanistic concerns around authenticity, value and significance in the negotiation of sustainable preservation outcomes. This corpus of knowledge contributes to specialized and non-specialized publications, promoting an overall understanding and appreciation of the cultural significance of heritage. The possibilities after digitalization are numerous. The only obstacle in this project are the conditions. Much more complicated parts can be scanned and processed if the appropriate equipment is available. But the initial goal has been achieved. Develop a methodology that is an example of reversible engineering for wood carvings. What were the steps of this methodology? Find a carving you think should be 'saved', digitalize it with the tools mentioned above, 'clean' the carving in one of the mentioned programs (although there are more on the market, another can be used), transfer the section to appropriate CAD/CAM programs and 'leave' the 3D printer and/or CNC machine to do the 'magic'. Although this methodology can be seen as type of business, it is also of great historical and cultural importance, a fusion of engineering with art.

\section{Notice}

The paper was presented at MOTSP $2021-12^{\text {th }}$ International Conference Management of Technology - Step to Sustainable Production, which took place in Poreč/Porenzo, Istria (Croatia), on September 8-10, 2021. The paper will not be published anywhere else.

\section{REFFERENCES}

[1] Kochov, A. (2006). 3D - Engineering, internal script. Faculty of Mechanical Engineering Skopje. (in Macedonian)

[2] Raja, V. \& Ferandes, K. (2012). Reverse Engineering, an Industrial Perspective, Springer, UK.

[3] https://www.researchgate.net/figure/The-smart-culturalheritage-concept_fig1_328301383
[4] Kochov, A. (2016). Technology of rapid prototyping, models and tools. UKIM. (in Macedonian)

[5] Orr, T. (2019). Rapid Prototyping: Increasing Proficiency in Design. Available: https://www.lumitex.com/blog/rapidprototyping

[6] Jamie, D. (2018). 3D Printing vs CNC Machining: Which is best for prototyping? Available: https://www.3dnatives.com/ en/3d-printing-vs-cnc-160320184/

[7] Coxworth, B. (2019). 3D-printing breakthrough paves the way for printed "wooden" products. Available: https://newatlas.com/wood-nanocellulose-3d-printing/60347/

[8] https://dveri.mk/en/

[9] NextEngine $3 D$ Scanner Tutorial. Available: https://wp.stolaf.edu/it/nextengine-3d-scanner-tutorial/

[10] Zagar, B. (2003). Reverse development of 3-D models using NURBS geometry, Ljubljana. (in Slovenian)

[11] Chambers, J. (2021). 10 Best 3D Scanner Apps in 2021 (Android \& iPhone). Available: https://all3dp.com/2/5-best3d-scanner-apps-for-your-smartphone/

[12] https://williamstownart.org/

[13] allthat3d. (2021). 3D Printer Price - How Much Does a 3D Printer Cost? Available: https://www.allthat3d.com/3dprinter-cost/

\section{Authors' contacts:}

\section{Atanas Kochov, PhD}

(Corresponding author)

Faculty of Mechanical Engineering - Skopje,

Karposh II bb, 1000 Skopje, Republic of North Macedonia

+389(0)71-299-299, atanas.kochov@mf.edu.mk

Lidija Stoleska, BSc

Faculty of Mechanical Engineering - Skopje, Karposh II bb, 1000 Skopje, Republic of North Macedonia +389(0)70-400-680, lidijastoleska99@gmail.com 